\title{
The Development and Optimization of Primer Sets Used to Study the Relative Expression of Androgen Receptor Gene in Turkey (Meleagris gallopavo)
}

\author{
Herjuno Ari Nugroho ${ }^{1 *}$, Rini Widayanti ${ }^{2}$, Tri Wahyu Pangestiningsih ${ }^{3}$, and Eli Supriyani ${ }^{2}$ \\ ${ }^{1}$ Research Center for Biology, Indonesian Institute of Sciences (LIPI), Jl. Jakarta-Bogor km 46, \\ Cibinong, West Java, Indonesia 16911. \\ ${ }^{2}$ Department of Biochemistry, Faculty of Veterinary Medicine, Universitas Gadjah Mada, Jl. Fauna \\ 02, Caturtunggal, Depok, Sleman, Yogyakarta, Indonesia 55281 \\ ${ }^{3}$ Department of Anatomy, Faculty of Veterinary Medicine, Universitas Gadjah Mada, Jl. Fauna 02, \\ Caturtunggal, Depok, Sleman, Yogyakarta, Indonesia 55281
}

\begin{abstract}
The Androgen Receptor (AR) Gene's expression is essential during puberty and testes maturation, which is also used as a reference for Turkey's breeding program. Therefore, this study aims to develop and optimize two primer sets for studying relative expression using qPCR technology. The primers were designed to amplify specific regions in the AR gene as the main target, and the $\beta$-actin gene as an internal control. They were tested using in-silico and amplicon sequencing, as well as efficiency calculation with a constructed standard curve from serially diluted reactions. Based on the sequencing methods, the primers amplified the corresponding regions of the respective targets. The primer for AR gene had an efficiency of $98.03 \%$, a slope of -3.37 , and an $\mathrm{R}^{2}$ of 0.995 , while that of the $\beta$-actin gene had an efficiency of $98.01 \%$, a slope of -3.371 , and an $\mathrm{R}^{2}$ of 0.999 . The two efficiencies exceeded the standard (93-103\%) value and the melting curve analysis showed that no non-specific amplification was discovered for both primers. According to the tests, the primers are suggested as acceptable to be used for the relative expression study of the AR gene in Turkey.
\end{abstract}

Keywords : Androgen Receptor Gene, Primer, optimization, Turkey

\section{Introduction}

Turkey has become a potential protein source popularly used in the USA and some European countries based on the higher protein and lower fat content compared to chicken $[1,2]$. Meanwhile in Indonesia, it is not optimally used as a protein source because of the high price and breeders' scarcity, and the meat is less consumed. Oktaviana et al. [2] analyzed turkey farming agribusiness management in Lampung Province and discovered that it was profitable.

\footnotetext{
* Corresponding author: herj001@gmail.com
} 
Reproduction and the need for good quality turkey, as broodstock are important aspects of the breeding program. Furthermore, several parameters were used for gobblers (adult male turkey) and jakes (yearling male turkey) selection, for instance, androgen hormones and its receptor's 'analysis [3]. AR gene's mRNA expression can be used as information about the gene regulation related to reproduction performances $[4,5]$. The AR primer is chosen since the Androgen only affects a target when it expresses enough receptors [6].

A relative expression study using quantitative PCR (qPCR) approaches requires specific primers which are a set of oligonucleotides that initiates amplification in the polymerase chain reaction. The primers need to be designed based on several considerable requirements, such as their length, primer pair's length difference, GC content, PCR product size, melting temperature, etc. [7]. Androgen receptor studies have been performed in turkey by Kiezun et al. $[4,5]$ which developed primer sets based on Gallus gallus transcripts. Therefore this study aims to develop and optimize the primers for studying a relative expression on M.gallopavo AR gene mRNA based on the bird's predicted transcripts.

\section{Materials and Method}

\subsection{Ethical Approval}

Every procedure conducted on the living animals used was approved by the Faculty of Veterinary Medicine, Universitas Gadjah Mada Ethical Clearance Committee, with document number: 0109/EC-FKH/Int./2019.

\subsection{Primer Design and In-Silico Test}

The primer sets were designed using Primer3Plus (http://bioinfo.ut.ee/primer3-0.4.0/), to amplify some areas in Meleagris gallopavo transcriptomes. AR gene's primer was given code AR which was designed to amplify the partial area of DNA-binding domain to Ligandbinding domain, from nucleotide number 167 to 393 of the gene's mRNA (Genbank: XM_031554642.1). $\beta$-actin gene's primer was given code BAK, which was designed to amplify the partial area in the gene's mRNA from nucleotide number 737 to 897 (Genbank: NM_001303179.1). The $\beta$-actin gene was chosen as internal control as it was also used by Kiezun, et.al [4,5]. The primers were designed according to Chuang, et.al. [7] and they were then submitted for an in-silico test for primer specificity using Primer-BLAST (https://www.ncbi.nlm.nih.gov/tools/primer-blast). Their sequences can be seen in Table 1 .

\subsection{Nucleic Acid Template Preparation}

The mRNA templates used were extracted from jakes' fresh testicle tissues $(n=2)$ collected from a slaughterhouse in Bantul Regency, Yogyakarta. The testicles were preserved in RNALater ${ }^{\mathrm{TM}}$ (ThermoFisher, USA) under $-20^{\circ} \mathrm{C}$ condition. Total RNA was extracted from each sample using Total RNA Mini Kit (Tissue) (Geneaid®, Taiwan). Its quality and quantity were examined using microplate reader Infinite ${ }^{\circledR} 200$ PRO NanoQuant (Tecan, Switzerland) and agarose gel electrophoresis respectively. About $1 \mu \mathrm{g}$ of the Total RNA was used as a template to synthesize cDNA using ReverTra Ace -a- with gDNA remover (Toyobo, Japan). The genomic DNA needs to be digested before cDNA synthesis since it potentially alters amplification reaction, leading to unspecific amplicons. 
Table 1. Primer sequences

\begin{tabular}{llccr}
\hline $\begin{array}{l}\text { Primer } \\
\text { Code }\end{array}$ & Sequence & Length & Target & Product Size \\
\hline AR & F: 5'- & 20 & AR gene & 227 \\
& AAGGTAAGCAGAAGTACCTG- & & & \\
& 3' & 20 & & \\
& R: 5'- & & & \\
& GTGTGTCATCACCATCTTAG-3' & & & \\
\hline BAK & F: 5'- & 20 & -actin & \\
& AGAGCTATGAACTCCCTGAT-3' & & gene & \\
& R: 5'- & 20 & & \\
& CTTACGGATATCCACATCAC-3' & & & \\
\hline
\end{tabular}

\subsection{Primer Specificity Test}

To clarify the primer sets' ability to amplify the target, the PCR products/amplicons from each primer set were submitted for sequencing. The conventional polymerase chain reaction was performed on two samples using a thermal cycler (Infinigen Biotech. Inc, USA). This was performed for both primer sets in 35 cycles of denaturation at $94^{\circ} \mathrm{C}$ for 30 seconds, annealing at $58^{\circ} \mathrm{C}$ for 30 seconds, and elongation at $72^{\circ} \mathrm{C}$ for 30 seconds. Furthermore, the amplicons were submitted to First Base Laboratory (Selangor, Malaysia) for Sanger Sequencing. The sequences produced were then aligned with reference sequences in Genbank by using Nucleotide BLAST (https://blast.ncbi.nlm.nih.gov/).

\subsection{Serial Dilution for Primers and cDNA Templates}

Serial dilution was performed on both primer sets and cDNA templates in three levels, using RNase/DNase-free water. The primers were diluted into three levels of concentration, namely $0.4 \mu \mathrm{M}, 0.2 \mu \mathrm{M}$, and $0.1 \mu \mathrm{M}$. The Templates were also diluted $1 \mathrm{x}(10 \mathrm{ng}), 4 \mathrm{x}(2.5 \mathrm{ng})$ and $10 \mathrm{x}$ (1ng).

\section{6. qPCR Reaction and Data Analysis}

Amplification for the qPCR reaction was performed using SYBRGreen ${ }^{\circledR}$ (Invitrogen, USA) as general fluorescence dye in AriaMx Real-Time PCR System (Agilent, USA). The PCR was conducted on 40 cycles of denaturation at $94^{\circ} \mathrm{C}$ for 30 seconds, annealing at $58^{\circ} \mathrm{C}$ for 30 seconds and lastly, elongation at $72^{\circ} \mathrm{C}$ for 30 seconds during which quantification data were collected. This amplification reaction was ended with a melting curve analysis at $65^{\circ} \mathrm{C}$ for 30 seconds. Threshold, primer efficiency, $\mathrm{R}^{2}$, slope and intercept values were set and calculated automatically using AriaMX's software.

\section{Result and Discussion}

\subsection{Primer Test}


The in-silico test performed using PrimerBLAST showed the AR and BAK primers annealed to the corresponding targets in Meleagris gallopavo AR gene (Genbank: XM_031554642.1) and $\beta$-actin gene (Genbank: NM_001303179.1) respectively. The AR primer set was also annealed to the AR gene of Gallus gallus (Genbank: NM_001040090.1). Since the two birds are genetically related, the sequences are probably conserved as stated by Katoh, et al. [8]. The AR primers used in this study were designed to amplify the partial area in M.gallopavo predicted AR transcripts. Meanwhile, Kiezun et al. [4] designed their primer based on Gallus gallus AR mRNA (Genbank: NM_001040090).

The PCR products/amplicons from 2 samples were run on $1.2 \%$ agarose gel electrophoresis and distinct bands were shown for each primer, as can be seen in Figure 1. These were about 200-300bp and 100-200bp for AR and BAK primers respectively. According to NucleotideBLAST, the sequences obtained from sequencing also had high similarity with M. gallopavo AR and $\beta$-Actin genes (Table 2). The BAK primer amplicons had $100 \%$ sequence similarity with the reference sequence (Genbank: NM_001303173), meanwhile, that of the AR primer had 98.34-100\% similarity (Genbank: XM_031554642.1).

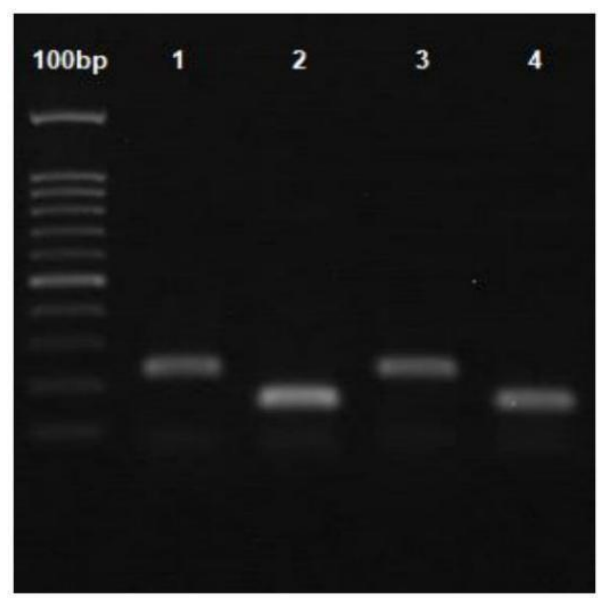

Fig 1. Gel electrophoresis for amplicons obtained using AR primer ( $1 \& 3)$ and BAK primer $(2 \& 4)$. Distinct bands were observed in appropriate sizes for each amplicon as well as unspecific bands.

Table 2. NucleotideBLAST on amplicons from both primer sets

\begin{tabular}{lllcc}
\hline Sample & Target & Primer & $\begin{array}{l}\text { Sequence similarity } \\
\text { with M.gallopavo } \\
\text { references }\end{array}$ & $\begin{array}{l}\text { Accession code for the } \\
\text { references (Genbank) }\end{array}$ \\
\hline $\mathbf{1}$ & AR & AR F & $98,9 \%$ & XM_019618131.1 \\
& & AR R & $99,48 \%$ & XM_019618131.1 \\
& \multirow{2}{*}{ B Actin } & BAK F & $100 \%$ & NM_001303173 \\
& & BAK R & $100 \%$ & NM_001303173 \\
$\mathbf{2}$ & AR & AR F & $98,34 \%$ & XM_019618131 \\
& \multirow{3}{*}{ A Actin } & AR R & $100 \%$ & XM_019618131 \\
& BAK F & $100 \%$ & NM_001303173 \\
& & BAK R & $100 \%$ & NM_001303173 \\
\hline
\end{tabular}




\subsection{Primer Concentration Optimization}

Three different concentrations of primer sets, namely $0.4 \mu \mathrm{M}, 0.2 \mu \mathrm{M}$, and $0.1 \mu \mathrm{M}$ were used in the qPCR reaction. Non-template control (NTC) was also included in the reaction as a negative control. Primer sets with concentrations of $0.4 \mu \mathrm{M}$ and $0.2 \mu \mathrm{M}$ produced close CT values compared to $0.1 \mu \mathrm{M}$, as can be seen in Figure 2 and Table 3. The CT values obtained during the concentration optimization ranged from 22.85 to 27.23 for AR primer and 15.89 to 20.83 for BAK primer.

Melting curve analysis showed a single peak for each sample, which indicated the qPCR reaction produced a specific single band only. The melting temperature for AR and BAK primers' PCR products were around $82.5-83^{\circ} \mathrm{C}$ and $88^{\circ} \mathrm{C}$ respectively. Also, the melting curve and Tm value can be seen in Figure 3 and Table 3 respectively. The same and close Tm value indicates the PCR products have similar sequences that tend to melt in the same temperatures [9]. The NTC samples had a CT value recorded as 40.2, and a Tm value of 74 . Since they had no added template, the amplification process occurred probably from primer dimer (PD) formation. This was proven in the melting curve analysis by having different $\mathrm{Tm}$ values with the test samples. Bustin et al. [10] stated that any CT value $>40$ generally need not be reported.

According to the reaction, the primer with a concentration of $0.1 \mu \mathrm{M}$ had the best result as indicated by the CT value. Furthermore, the CT values produced were around 20.83 and 27.23 for AR and BAK primers respectively. Kuang et al. [9] stated CT values used for a relative expression study need to be around 20-30.

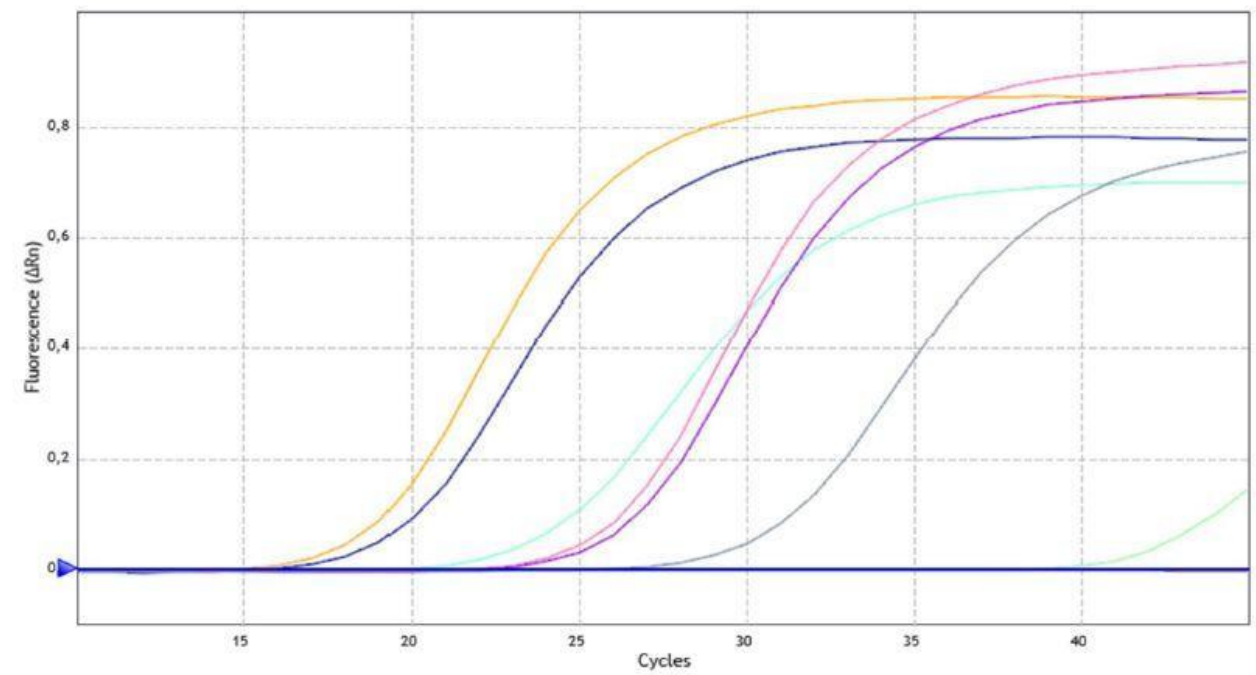

H1, 0.4 uM beta actin primer R5 sample H4, 0.4 uM AR primer R5 sample

H2, 0.2 un beta actin primer R5 sample

H5, 0.2 uM AR primer R5 sample

$\mathrm{H} 8,0.4$ uM beta actn primer NTC

Fig 2. Optimization of $\mathrm{AR}$ and $\mathrm{BAK}$ primers using a serial dilution, namely $0.4 \mu \mathrm{M}, 0.2 \mu \mathrm{M}$ and 0.1 $\mu \mathrm{M}$. The primer with $0.4 \mu \mathrm{M}$ and $0.2 \mu \mathrm{M}$ concentrations produced a closer $\mathrm{CT}$ value compared to $0.1 \mu \mathrm{M}$. 


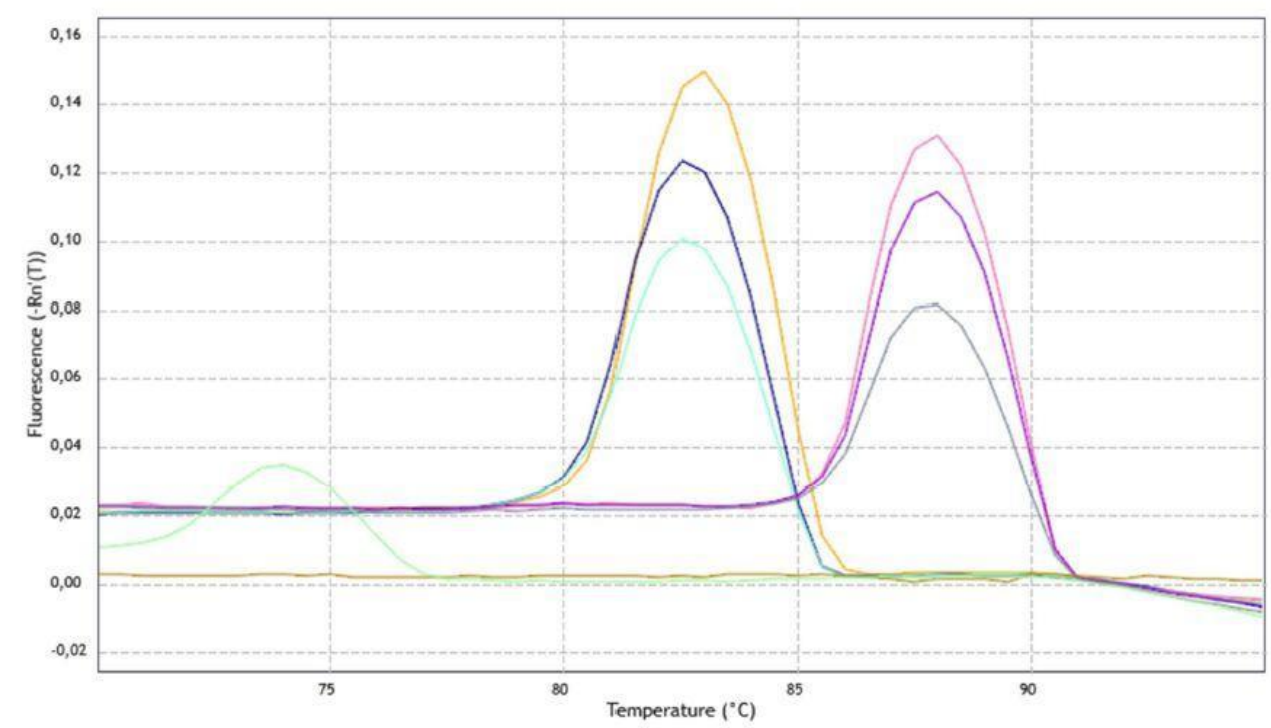

H1, 0.4 un beta actin primer $\mathrm{R} 5$ sample $\mathrm{H2}, 0.2$ un beta actin primer $\mathrm{R} 5$ sample $\mathrm{H} 3,0.1$ un beta actin primer $\mathrm{R} 5$ sample $\mathrm{H4}, 0.4$ un AR primer $\mathrm{R} 5$ sample - H5, 0.2 uM AR primer R5 sample $\mathrm{H6}, 0.1$ uM AR primer R5 sample

Fig 3. Melting curve analysis on primer optimization reaction. The curve showed a single peak for each reaction indicating the $\mathrm{qPCR}$ reaction produced a specific single amplicon.

Table 3. CT and Tm values for each sample

\begin{tabular}{lccc}
\hline Well & Label & $\begin{array}{l}\text { CT/Cq } \\
(\Delta \mathbf{R n})\end{array}$ & Product Tm 1 (-Rn'(T)) \\
\hline H1 & $0.4 \mu \mathrm{M} \mathrm{BA}$ & 15.89 & 83 \\
H2 & $0.2 \mu \mathrm{M} \mathrm{BA}$ & 16.68 & 82.5 \\
H3 & $0.1 \mu \mathrm{M} \mathrm{BA}$ & 20.83 & 82.5 \\
H4 & $0.74 \mu \mathrm{M} \mathrm{AR}$ & 2285 & 88 \\
H5 & $0.2 \mu \mathrm{M} \mathrm{AR}$ & 23.23 & 88 \\
H6 & $0.1 \mu \mathrm{M} \mathrm{AR}$ & 27.23 & 88 \\
H7 & & - & 70.5 \\
H8 & NTC & 40.02 & 74 \\
\hline
\end{tabular}

\subsection{AR Primer Reaction on Serially Diluted Template}

The primer set was attempted to amplify three levels of serial dilution, 1/1, 1/3, and 1/9, aimed at efficiency calculation. In addition, the dilution and CT value were directly proportional as higher dilution caused the template concentration to become lower. The amplification curve and CT value can be seen in Figure 4 and Table 4. Melting curve analysis was also performed to clarify that the amplification produces a specific amplicon. The melting temperature on three levels of serial dilution showed the same Tm value, indicating the dilutions' amplifications were structurally similar. The melting curve and Tm values were are presented in Figure 5 and Table 4 respectively. 


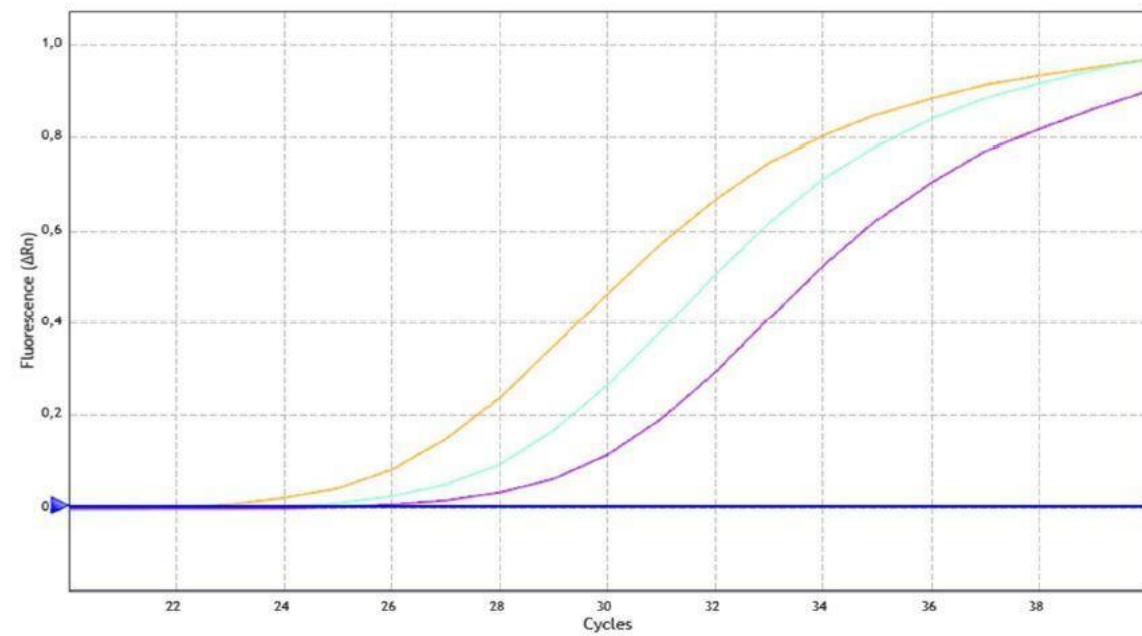

G1, AR primer, original conc. R1 sample $O$ G2, AR primer, 1/3 R1 sample

G3, AR primer, 1/9 R1 sample

Fig 4. The curve obtained from qPCR with AR primer and serially diluted templates. The dilutions were $1 / 1,1 / 3$, and $1 / 9$, with an initial concentration of $10 \mathrm{ng} / \mu 1$. The lower concentration tended to produce a higher $\mathrm{CT}$ value.

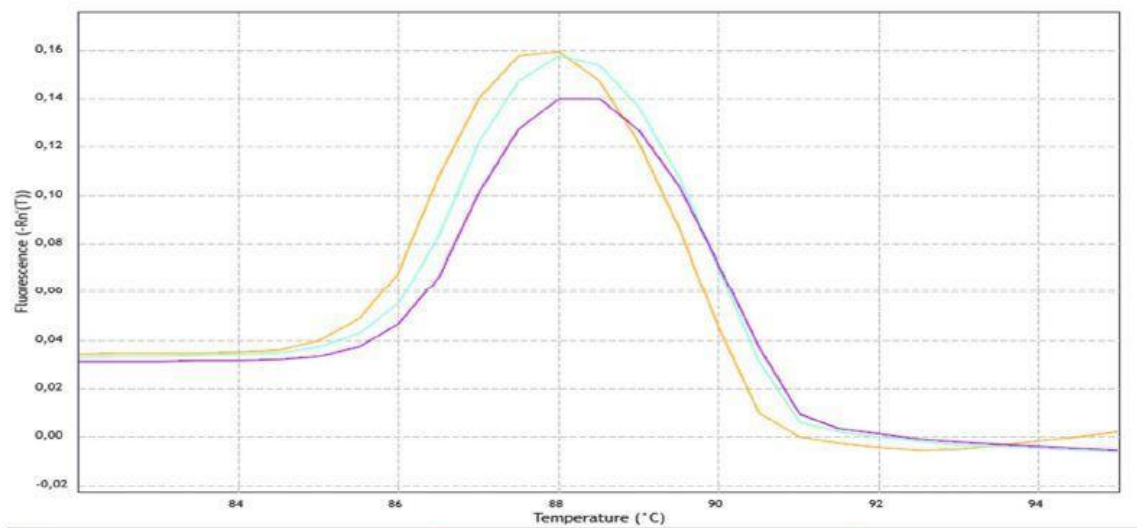

G1, AR primer, original conc. R1 sample $\bigcirc$ G2, AR primer, 1/3 R1 sample

G3, AR primer, 1/9 R1 sample

Fig 5. Melting curve analysis on qPCR with AR primer and serially diluted templates. The reaction's curve showed an almost similar single peak, indicating that all the serially diluted templates produced a similar specific amplicon.

Table 4. Calculation of $\mathrm{CT}$ and $\mathrm{Tm}$ values for $\mathrm{qPCR}$ with $\mathrm{AR}$ primer and serially diluted templates

\begin{tabular}{clcc}
\hline well & Label & CT/Cq $(\Delta \mathbf{R n})$ & Product Tm 1 (-Rn'(T)) \\
\hline G1 & AR 1/1 & 23.18 & 88 \\
G2 & AR 1/3 & 24.99 & 88 \\
G3 & AR 1/9 & 26.4 & 88 \\
\hline
\end{tabular}

A standard curve was constructed to calculate the primer efficiency and can be seen in Figure 6. It produced an efficiency of $98.03 \%$, a slope of -3.37 , and an $\mathrm{R}^{2}$ of 0.995 . Kuang 
et.al. [9], recommend that the acceptable primer needs to have an efficiency of 93\%-103\%, a slope of -3.2 to -3.5 , and an $\mathrm{R}^{2}$ of more than 0.98 . However, the AR primer set used exceeded the recommended one.

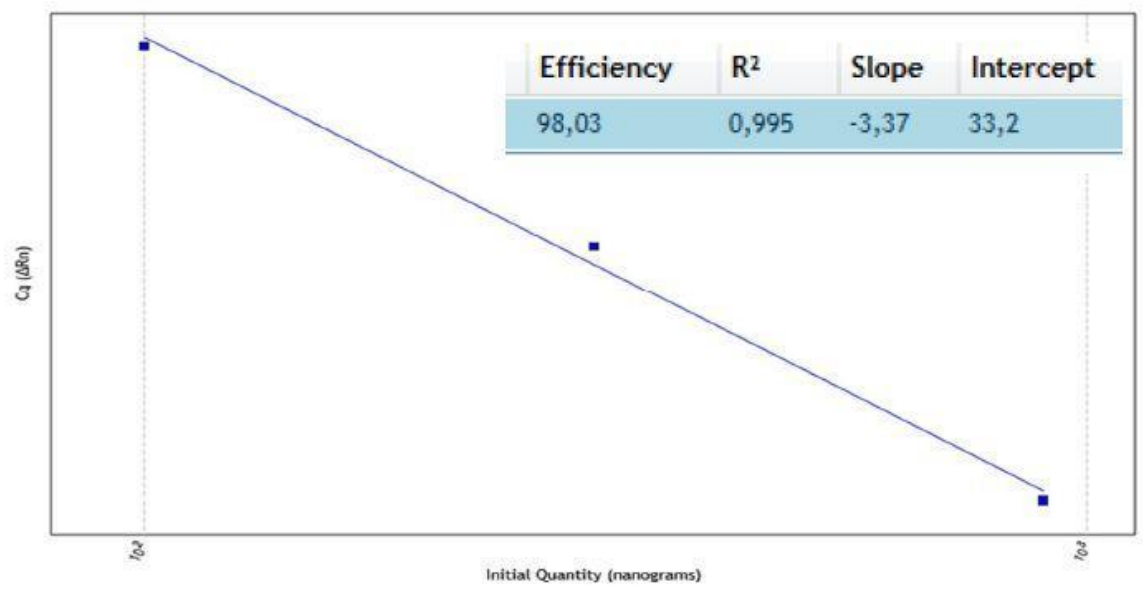

Fig 6. The standard curve constructed from $\mathrm{qPCR}$ reaction with AR Primer and serially diluted templates. It produced an efficiency of $98.03 \%$, a slope of -3.37 and an $\mathrm{R}^{2}$ of 0.995 .

\subsection{BAK Primer Reaction on Serially Diluted Template}

Primer efficiency test was also performed on BAK Primer. Furthermore, the reaction was conducted on three levels of serial dilution, 1/1,1/3, and 1/9, with an initial concentration of $10 \mathrm{ng} / \mu \mathrm{l}$. This produced a similar pattern with AR primer, where higher dilution produced higher CT value, while the amplification curve can be seen in Figure 7.

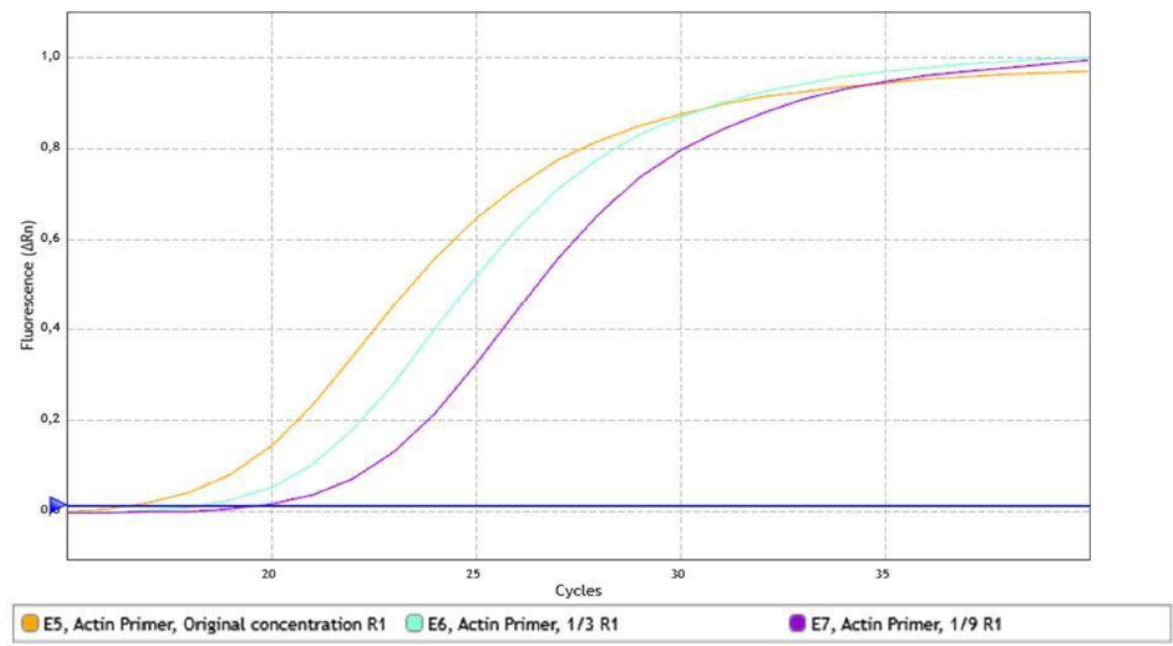

Fig 7. The curve obtained from qPCR with BAK primer and serially diluted templates. The dilutions were $1 / 1,1 / 3$, and $1 / 9$, with an initial concentration of $100 \mu \mathrm{g} / \mu \mathrm{l}$. The lower concentration tended to produce a higher CT value. 
The melting curve analysis on three levels of serial dilution templates also produced a single peak. This indicated that the amplicons from the serially diluted samples were structurally similar as they melt at the same temperature. The result of the analysis is presented in Figure 8.

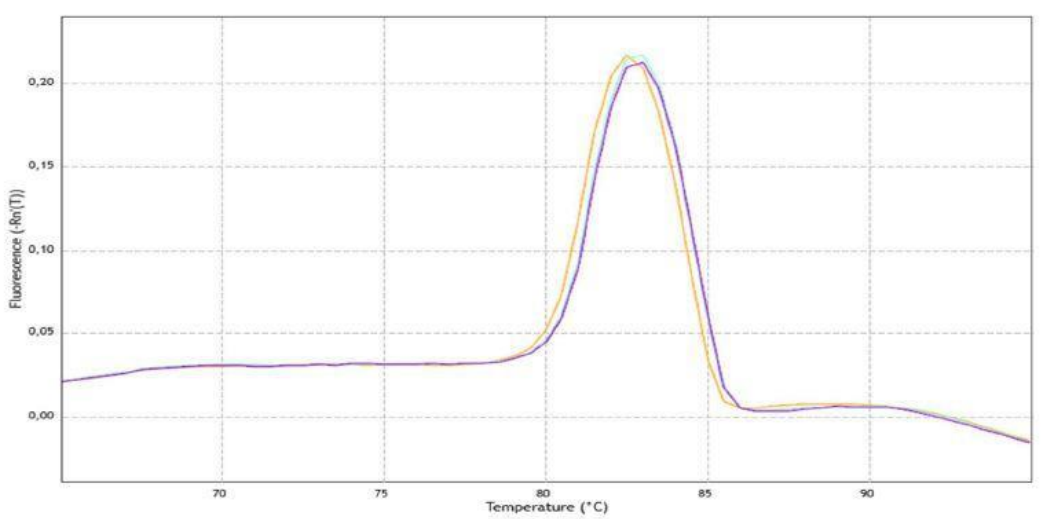

E5, Actin Primer, Original concentration R1 E6, Actin Primer, 1/3 R1

E7, Actin Primer, 1/9 R1

Fig 8. The melting curve analysis on $\mathrm{qPCR}$ with BAK primer and serially diluted templates. The reaction's curve showed a similar single peak, which indicated all the serially diluted templates produced similar specific amplicon.

A standard curve was also constructed to calculate BAK primer efficiency. It produced an efficiency of 98.01, a slope of -3.371 , and an $\mathrm{R}^{2}$ of 0.999 as can be seen in Figure 9. The BAK primer also exceeded the minimal recommendation stated by Kuang et al. [9].

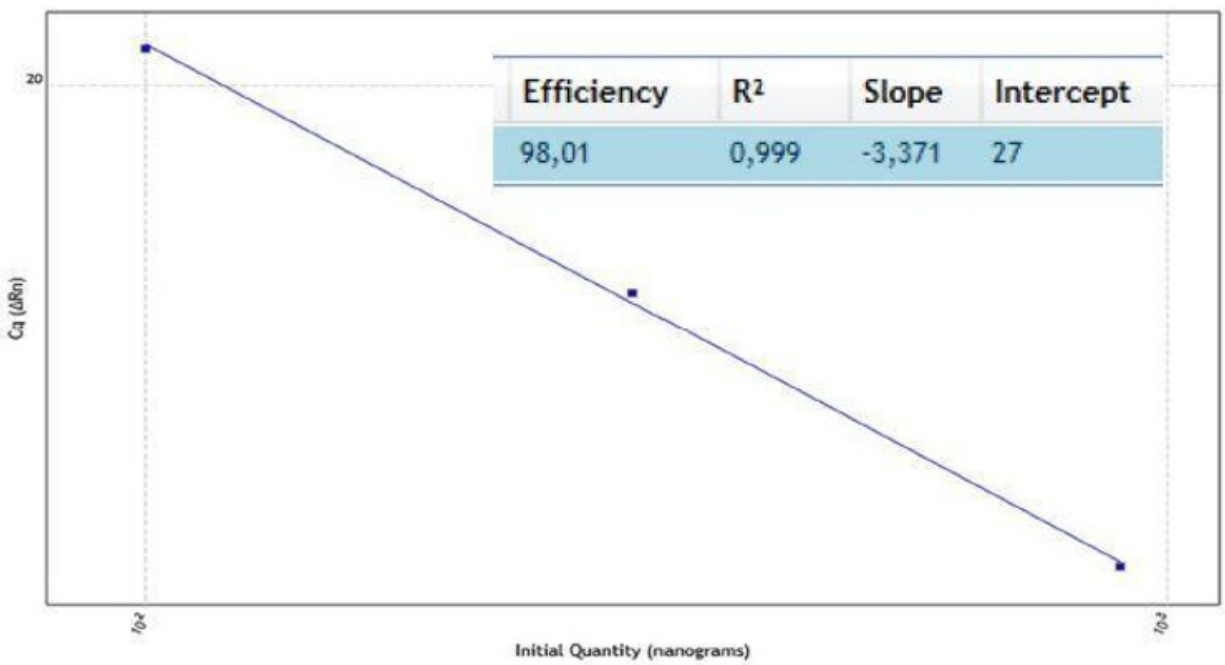

Fig 9. A standard curve constructed from $\mathrm{qPCR}$ reaction with BAK Primer and serially diluted templates. It produced an efficiency of 98.01 , a slope of -3.371 , and an $\mathrm{R}^{2}$ of 0.999 .

The AR and BAK primer sets designed in this research amplified the AR and $\beta$-Actin genes, respectively. These were specific to anneal on the target sequences as proven by in- 
silico and amplicon sequencing followed by nucleotide BLAST. They also annealed on closely related organisms as shown in G. gallus gene by the in-silicon method. The amplification reaction conducted using both primers produced a specific amplicon as shown by the melting curve analysis. This occurred in NTC samples after the $40^{\text {th }}$ cycle and can be eliminated from the analysis [10]. The two above-mentioned sets exceeded the minimum primer efficiency parameters stated by Kuang et al [9].

\section{Conclusion}

The primer sets developed exceeded the minimum requirement. They need to be tested on many samples and in different age groups to distinguish genes' expression.

The authors are grateful to Universitas Gadjah Mada for funding this study through the 2018 RTA Program.

\section{References}

1. A. Bordoni, F.Danesi. (Elsevier Ltd., 2017).

2. E. Oktaviana, D.A.H.Lestari, Y.Indriani. JIIA. 4, 3 (2016)

3. R.A.Dornas, A.G.Oliviera, M.O.Dias, G.A.Mahecha, C.A.Oliviera. Gen.Comp.Endocrinol. 166 (2008).

4. J.Kiezun, A.Leska, B.Kaminska, J.Jankowski, L.Dusza. Gen. Comp. Endocrinol. 214 (2015)

5. J. Kiezun, B.Kaminska, J.Jankowski, L. Dusza. Gen. Comp. Endocrinol, 217-218 (2015)

6. R.A.Davey, M.Grossmann Clin Biochem Rev. 37, 1 (2016)

7. L.Y.Chuang, Y.H.Cheng, C.H.Yang, S. Biotechnol Lett., 35, 10 (2013)

8. H.Katoh, Y.Ogino, G.Yamada, FEBS Letter. 580, 6 (2006)

9. J.Kuang, X.Yan, A.J.Genders, C.Granata, D.J.Bishop, Plos One 13, 5 (2018)

10. S.A.Bustin, V.Benes, J.A.Garson, J.Hellemans, J.Huggett, M.Kubista, R.Mueller, T.Nolan, M.W.Pfaffl, G.L.Shipley, J.Vandesompele, C.T.Wittwer. Clin.Chem 55, 4 (2009) 